\title{
nab-Paclitaxel for the treatment of pancreatic cancer
}

\author{
This article was published in the following Dove Press journal: \\ Cancer Management and Research \\ 16 March 2017 \\ Number of times this article has been viewed
}

\section{George Kim}

2 Ist Century Oncology, University of Florida Health Oncology, Jacksonville, FL, USA
Correspondence: George Kim 2 Ist Century Oncology, University of Florida Health Oncology, 775 I Baymeadows Road E, Ste 205, Jacksonville, FL 32256, USA Email gmrpkim@gmail.com
Background: Nanoparticle albumin-bound paclitaxel ( $n a b-\mathrm{P})$ plus gemcitabine (Gem) became a standard treatment option for metastatic pancreatic cancer (MPC) following positive results from a global phase III trial (MPACT). A large number of studies have now published results on the use of $n a b$-P/Gem to treat advanced and early-stage disease, warranting a comprehensive review. The main goal of this systematic review is to summarize the efficacy and safety data of $n a b-\mathrm{P} / \mathrm{Gem}$ for the treatment of pancreatic cancer (PC).

Methods: This systematic review includes results from studies that either published results in a peer-reviewed journal or presented the results at a major oncology conference.

Results: Sixty-two studies were included (50 in the advanced/metastatic setting and 12 in the locally advanced setting). Most studies on the treatment of MPC were exclusively first line $(33 / 50)$. Nevertheless, the studies in this review comprised a broad spectrum of patients, including those $<65$ and $\geq 65$ years of age and those with a Karnofsky performance status of 70-100. Median overall survival (OS) in studies of $n a b-\mathrm{P} / \mathrm{Gem}$ in the advanced/metastatic setting ranged from 8.7 to 13.5 months. In addition, 15 studies of patients with advanced/metastatic PC examined $n a b-\mathrm{P} / \mathrm{Gem}$ as a backbone on which to add a variety of agents, including cancer stem cell inhibitors, stromal disrupting agents, and immune-modulating agents (median OS, 6.9-17 months). Ongoing trials are investigating $n a b-\mathrm{P} / \mathrm{Gem}$ with or without other agents across disease settings.

Discussion: Studies conducted after MPACT have demonstrated that $n a b-\mathrm{P} / \mathrm{Gem}$ is an effective regimen for the first-line treatment of MPC for a wide range of patients. Regimens using $n a b-\mathrm{P} / \mathrm{Gem}$ as a backbone on which to combine additional agents are being studied actively, particularly in the advanced disease setting. Ongoing studies will yield valuable insights on the utility of nab-P-containing regimens to improve patient outcomes in PC in both earlier-stage and advanced disease.

Keywords: pancreatic cancer, nab-paclitaxel, metastatic, neoadjuvant, systematic review

\section{Introduction}

More than 50,000 new pancreatic cancer (PC) cases and >40,000 cancer-related mortalities due to PC are expected in the USA in 2016. ${ }^{1,2}$ The 5-year survival rate for all stages of PC combined is $8 \%$. Although those with resectable disease have a more favorable prognosis ( 5 -year survival $\approx 29 \%$ ), $\approx 52 \%$ of patients are diagnosed with metastatic disease, which confers a less favorable outlook ( 5 -year survival $\approx 3 \%)$. ${ }^{2}$ Since the approval of gemcitabine (Gem) in 1997, no phase III trial in advanced/metastatic disease had demonstrated a clinically and statistically significant improvement in overall survival (OS) over Gem alone ${ }^{3}$ until recently. The treatment landscape for metastatic 
disease has evolved to include 2 key regimens: folinic acid, 5-fluorouracil, irinotecan, and oxaliplatin (FOLFIRINOX) and nanoparticle albumin-bound paclitaxel (nab-P) plus Gem (nab-P/Gem). The FOLFIRINOX regimen was approved based on a French multicenter phase II/III trial that reported significant improvements in OS with FOLFIRINOX versus Gem (median, 11.1 vs 6.8 months; hazard ratio [HR], 0.57; $P<0.001$ ), but significant adverse events were also observed. ${ }^{4}$ The $n a b$-P/Gem regimen was approved in many countries after the phase III MPACT trial demonstrated that the addition of $n a b^{\circledR}-\mathrm{P}$ (Abraxane ${ }^{\circledR}$; Celgene Corporation, Summit, NJ, USA) to Gem improved OS versus Gem (median, 8.7 vs 6.6 months; HR, 0.72; P<0.001). ${ }^{5}$ Currently, the National Comprehensive Cancer Network (NCCN) and European Society for Medical Oncology (ESMO) recommend treatment with FOLFIRINOX or $n a b$-P/Gem as standards of care for patients with metastatic pancreatic cancer (MPC). ${ }^{6,7}$ Age, performance status (PS), and other clinical factors are considered when deciding which regimen to use; Gem monotherapy is currently reserved for patients ineligible to receive combination chemotherapy. ${ }^{6}$

$n a b$-P/Gem and FOLFIRINOX have not been approved for earlier-stage disease; however, numerous trials are exploring their utility. The NCCN recommends chemotherapy for unresectable locally advanced PC (LAPC) and chemoradiation for selected patients, preferably after induction chemotherapy for tumor control. ${ }^{6}$ Currently, no clear evidence exists to support the use of $n a b-\mathrm{P} / \mathrm{Gem}$ over FOLFIRINOX or vice versa, and several trials are investigating their efficacy and safety. ${ }^{8}$

A population-based study of $>3,000$ patients showed that $n a b-\mathrm{P} / \mathrm{Gem}$ is the most commonly used chemotherapy regimen for the first-line treatment of MPC in the USA, ${ }^{9}$ possibly due to the toxicity profile of FOLFIRINOX, which limits its use to younger/fitter patients. The extensive use of $n a b-\mathrm{P} / \mathrm{Gem}$ in both academic and community settings coupled with $>100$ current and active clinical trials in PC warrants a comprehensive review of clinical data to gain a better understanding of how this regimen is being used for the treatment of PC and associated outcomes. The overall goal of this review is to summarize recent data regarding the safety and efficacy of regimens that include $n a b-\mathrm{P} / \mathrm{Gem}$ for patients with PC.

\section{Methods}

The search terms "nab-paclitaxel and (pancreatic or pancreas)" were entered in PubMed to retrieve publications from January 1, 2011 to June 30, 2016. Abstracts from the annual meetings of the American Society of Clinical
Oncology (ASCO) 2011-2016, the Gastrointestinal Cancers Symposium (ASCO GI) 2011-2016, the European Cancer Organisation/ESMO 2011-2015, the ESMO World Congress on Gastrointestinal Cancer 2015 and 2016, and the Italian Association of Medical Oncology (2014) were searched using the term "nab-paclitaxel." Clinical trials and institutional analyses of $n a b$-P in all stages of PC were included. Duplicates, electronic abstracts, case studies, cost studies, meta-analyses, and studies of the effects of eligibility criteria were excluded. The website www.clinicaltrials.gov was searched using the terms "nab-paclitaxel" OR "Abraxane" AND "pancreatic" AND "adenocarcinoma" to identify ongoing trials without results; only open, active, phase II-III trials with a sample size $\geq 100$ were included.

\section{Results}

\section{Studies of nab-P in advanced/ metastatic PC}

Fifty studies evaluating $n a b-\mathrm{P}$ in MPC were retrieved (Figure 1; Table 1). Approximately one-half were retrospective analyses. MPACT was the only phase III study, and all other prospective trials were phase I or II. Two-thirds of studies evaluated $n a b-\mathrm{P}$ in the first-line setting, and approximately one-third of those studies assessed $n a b$-P/Gem with an additional agent. $n a b$-P was most often evaluated at a dose of

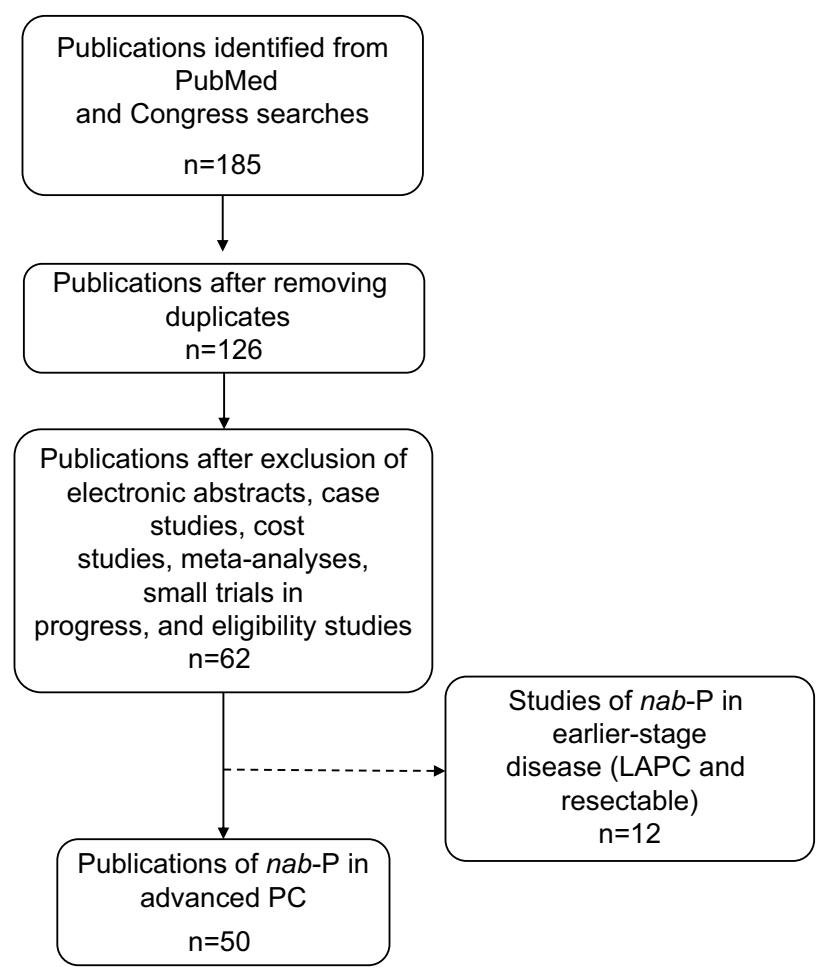

Figure I Schematic of method for systematically selecting studies for inclusion in the database.

Abbreviations: LAPC, locally advanced pancreatic cancer; nab-P, nanoparticle albumin-bound paclitaxel; PC, pancreatic cancer. 
Table I Characteristics of advanced/MPC studies $(n=50)$

\begin{tabular}{ll}
\hline Characteristic & $\begin{array}{l}\text { Number of } \\
\text { studies (\%) }\end{array}$ \\
\hline Study design & $\mathrm{I}(2)$ \\
Prospective & $9(18)$ \\
Pilot & $6(12)$ \\
Phase I & $10(20)$ \\
Phase I/II & $\mathrm{I}(2)$ \\
Phase II & $23(46)$ \\
Phase III & \\
Retrospective/institutional experience & \\
First-line only nab-P & \\
Regimen & $33(66)$ \\
nab-P/Gem & $29(58)$ \\
nab-P/Gem + other agent & $15(30)$ \\
nab-P + other agent & $3(6)$ \\
nab-P monotherapy & $3(6)$ \\
\hline
\end{tabular}

Abbreviations: Gem, gemcitabine; MPC, metastatic pancreatic cancer; nab-P, nanoparticle albumin-bound paclitaxel.

$125 \mathrm{mg} / \mathrm{m}^{2}$, which was given the first 3 of 4 weeks (qw 3/4). The tables in this systematic review cover MPC (Tables 1-3), neoadjuvant treatment or locally advanced disease (Table 4), and ongoing trials in all settings (Table 5).

\section{nab-P/Gem in MPC}

Ten studies reported the median OS for first-line $n a b-\mathrm{P} / \mathrm{Gem}$ in patients with advanced PC; ; $50-18$ Table 2 lists 8 of these studies with a population $>45$ patients. The most commonly used dose and schedule were those used in the MPACT trial: $n a b-\mathrm{P} 125 \mathrm{mg} / \mathrm{m}^{2}$ plus Gem $1,000 \mathrm{mg} / \mathrm{m}^{2}$ administered on a qw 3/4 schedule..$^{5,14,19,20}$ Patients treated with this dose and schedule experienced a median OS ranging from 8.7 to 13.5 months $^{5,18}$ and 1-year survival ranging from $35 \%$ to $62 \% .{ }^{5,14,19,20}$ Most prospective studies evaluating this dose and schedule were single-arm trials.

\section{nab-P/Gem in MPC - age}

It may be expected that younger patients would experience longer survival and improved tolerability compared with older patients. However, most studies, including MPACT, suggest that older patients benefit from $n a b-\mathrm{P} / \mathrm{Gem}$ in terms of efficacy without increased risk of toxicity. Approximately $40 \%$ of patients enrolled in MPACT were $>65$ years. ${ }^{5}$ Median OS was 9.6 and 7.7 months for patients $<65$ and $\geq 65$ years, respectively, and the toxicity profiles were similar between age groups. ${ }^{5}$ The combination in MPACT demonstrated significant OS benefit over Gem alone in both age groups: $<65$ years (HR, $0.65 ; P<0.001)$ and $\geq 65$ years (HR, $0.80 ; P=0.048$ ).

A study $(\mathrm{N}=37)$ including patients treated with first-line or $\geq$ second-line $n a b-\mathrm{P} / \mathrm{Gem}$ for MPC showed that OS was not significantly different between patients $\geq 66$ years and those $<66$ years of age (median, 10.5 vs 9 months; $P=0.49$ ). ${ }^{21}$ Similarly, a large Italian database review of patients $(\mathrm{N}=208)$ with advanced PC treated with $n a b-\mathrm{P} / \mathrm{Gem}$ demonstrated that age ( $\geq 75$ vs $<75$ years) was not significantly associated with efficacy or toxicity with respect to median OS

Table 2 Overall survival (OS) with first-line nab-P/Gem in studies of $\geq 45$ patients with metastatic pancreatic cancer

\begin{tabular}{|c|c|c|c|c|c|c|c|}
\hline First author, year & Type of study & $\operatorname{Agent}(s)^{a}$ & $\mathbf{n}$ & $\begin{array}{l}\text { Age, median, } \\
\text { years }\end{array}$ & PS & $\begin{array}{l}\text { Median OS }(95 \% \mathrm{Cl}) \text {, } \\
\text { months }\end{array}$ & $P$ value \\
\hline Von Hoff, $201 I^{10}$ & $\mathrm{Ph} \mathrm{I/II}$ & $n a b-\mathrm{P} / \mathrm{Gem}$ & 67 & $6 I^{c}$ & ECOG 0-I & $12.2^{\mathrm{c}}$ & NR \\
\hline \multirow[t]{3}{*}{ Cartwright, 2014" } & Retro & $n a b-P^{d} / G e m$ & 189 & Gem-based & Gem-based regimens: & 10.2 & NR \\
\hline & & Gem + other chemo & 1,567 & regimens: 70 & KPS $<70,7 \%$ & 7.0 & \\
\hline & & FOLFIRINOX & 666 & 60 & KPS $<70,1 \%$ & 11.2 & \\
\hline \multirow[t]{5}{*}{ Santoni, $2014^{12}$} & Retro & $n a b-P^{d} / G e m$ & 41 & 66 & NR & 11.6 & NR \\
\hline & & Gem & 159 & & & 5.5 & \\
\hline & & Gem + cisplatin/oxaliplatin & 234 & & & 7.5 & \\
\hline & & Gem + capecitabine & 43 & & & 9.1 & \\
\hline & & FOLFIRINOX & 101 & & & 13.0 & \\
\hline Krishna, $2015^{13}$ & Retro & $n a b-P / G e m e$ & 49 & 65 & ECOG 0-I & II.I (5.3-not reached) & NA \\
\hline MPACT & $\mathrm{Ph}$ III & nab-P/Gem & 431 & 62 & $\mathrm{KPS}<80, \approx 7 \%$ & $8.7(7.9-9.7)$ & $<0.001$ \\
\hline Goldstein, $2015^{5}$ & & Gem & 430 & 63 & KPS $<80,8 \%$ & $6.6(6.0-7.2)$ & \\
\hline Giordano, 2015 $5^{50}$ & Retro & $n a b-\mathrm{P} / \mathrm{Gem}$ & 208 & 67 & ECOG PS $2,17.8 \%$ & II (8.8-13.2) & NA \\
\hline Shen, $2016^{17}$ & $\mathrm{Ph} I I$ & $n a b-P / G e m$ & 83 & 57 & KPS 70-80, 30\% & $9.2(5.29-7.16)$ & NA \\
\hline \multirow[t]{2}{*}{ Hammel, $2016^{15}$} & $\mathrm{Ph}$ II & nab-P/Gem & 39 & 65.3 & ECOG 2, $15.4 \%$ & $9.2(6.0-13.6)$ & NR \\
\hline & & nab-P + sLV5FU2 & 75 & 66.2 & ECOG $2,16.0 \%$ & II.4 (8.8-16.6) & \\
\hline
\end{tabular}

Notes: a ${ }^{n} a b-\mathrm{P}$ at $125 \mathrm{mg} / \mathrm{m}^{2}$ the first 3 of 4 weeks (qw 3/4) unless otherwise indicated. ${ }^{b}$ nab-P at 100,125 , or $150 \mathrm{mg} / \mathrm{m}^{2} \mathrm{qw} 3 / 4$. 'For nab-P I25 mg/m² $\mathrm{qw} 3 / 4$ (n=44). ${ }^{\mathrm{d} D o s e}$ and schedule of nab-P not reported. enab-P at $125 \mathrm{mg} / \mathrm{m}^{2}$ and $\mathrm{Gem}$ at $\mathrm{I}, 000 \mathrm{mg} / \mathrm{m}^{2}$ both given $\mathrm{q} 2 \mathrm{w}$. Supportive care also included dexamethasone $12 \mathrm{mg} 30 \mathrm{~min}$ prior to chemotherapy administration. $\mathrm{f} n a b-\mathrm{P}$ at 100 or $125 \mathrm{mg} / \mathrm{m}^{2}$ qw $3 / 4$.

Abbreviations: chemo, chemotherapy; Cl, confidence interval; ECOG, Eastern Cooperative Oncology Group; FOLFIRINOX, folinic acid, 5-fluorouracil, irinotecan, and oxaliplatin; Gem, gemcitabine; KPS, Karnofsky performance status; NA, not applicable; NR, not reported; nab-P, nanoparticle albumin-bound paclitaxel; Ph, phase; PS, performance status; Retro, retrospective; sLV5FU2, simplified leucovorin and 5 -fluorouracil regimen. 
(11.4 vs 11 months; $P=0.86)$, disease control rate $(69 \%$ vs $61 \% ; P=0.64)$, grade $3 / 4$ neutropenia ( $25 \%$ vs $28 \%$ ), and neurotoxicity ( $9 \%$ vs $12 \%){ }^{22}$ Additionally, an exploratory analysis from MPACT showed that the percentages of patients requiring $n a b-\mathrm{P}$ dose reductions were similar between age groups ( $42 \%$ for patients $\geq 65$ years vs $40 \%$ for patients $<65$ years $).{ }^{23}$

\section{nab-P/Gem in MPC - PS}

Data on whether patients with a better PS might receive greater benefit from $n a b-\mathrm{P} / \mathrm{Gem}$ than patients with a poorer PS are inconclusive; however, similar to the literature in older patients, several studies suggest that less fit patients receive meaningful benefit from the regimen. Stratification of the MPACT population by Karnofsky PS (KPS) demonstrated significantly better OS in the fitter (KPS 90-100) versus less fit (KPS 70-80) group in the combination arm (median, 9.7 vs 7.6 months; HR, 0.76; $P=0.009$ ) and the Gem arm (median, 7.9 vs 4.3 months; HR, $0.57 ; P<0.001) .{ }^{5}$ In the KPS 70-80 subpopulation, $n a b-\mathrm{P} / \mathrm{Gem}$ extended median OS by $>3$ months compared with Gem alone (7.6 vs 4.3 months; HR, 0.59; $P<0.001)$.

A small phase I/II trial examined the effect of $n a b-\mathrm{P} /$ Gem in patients with an Eastern Cooperative Oncology Group (ECOG) PS of $2 .{ }^{24}$ The results of the phase I portion suggest that these patients were able to receive the standard dose of $n a b-\mathrm{P} / \mathrm{Gem}$; the relative dose intensity was $100 \%$ in 6 patients who received nab-P $125 \mathrm{mg} / \mathrm{m}^{2}$ plus Gem $1,000 \mathrm{mg} / \mathrm{m}^{2}$ qw 3/4.

In a retrospective analysis of 39 patients with unresectable LAPC or MPC treated with $n a b-\mathrm{P} / \mathrm{Gem},{ }^{25}$ patients with an ECOG PS of 1 survived longer than patients with an ECOG PS of 2 (median OS, 15 vs 7 months; $P=0.032$ ). ${ }^{25}$ Similarly, the previously mentioned Italian retrospective analysis of patients with advanced $\mathrm{PC}(\mathrm{N}=208)$ treated with $n a b-\mathrm{P} / \mathrm{Gem}$ showed a numerically shorter OS in the ECOG PS 2 versus ECOG PS 0-1 group (median, 8.7 vs 11.2 months; $P=0.07$ ), but the difference was not significant. ${ }^{22}$ In addition, toxicities did not appear to be influenced by PS, because similar percentages of patients with PS $0-1$ and PS 2 developed neutropenia (31\% and 34\%, respectively) and neurotoxicity (17\% in each group). Collectively, these studies suggest that, although PS may affect OS, nab-P/Gem seems to be effective regardless of PS.

nab-P/Gem in MPC - real-world comparative effectiveness studies

Although clinical trials comparing $n a b-\mathrm{P} / \mathrm{Gem}$ with FOLFIRINOX for the treatment of $\mathrm{PC}$ have not yet reported results, retrospective analyses have explored these standard-of-care regimens with one another and/or Gem for the treatment of MPC. ${ }^{11,12,26-29}$ One study reported a median OS of 10.2 months with $n a b-\mathrm{P} / \mathrm{Gem}(\mathrm{n}=189)$ versus 11.2 months with FOLFIRINOX $(n=666)$ and 7 months for Gem combined with other chemotherapies $(n=1,567) .{ }^{11}$ Similar results were reported from another retrospective analysis: median OS of 11.6 months with $n a b-\mathrm{P} / \mathrm{Gem}(\mathrm{n}=41)$ versus 13 months with FOLFIRINOX $(\mathrm{n}=101)$ and 7.5-9.1 months for Gem plus other chemotherapies $(n=277) .{ }^{12}$ A real-world analysis based on electronic medical records of patients $(\mathrm{N}=202)$ receiving first-line treatment for advanced PC demonstrated similar comparative effectiveness for $n a b-\mathrm{P} /$ Gem versus FOLFIRINOX (database persistence [proxy for OS], median, 8.6 months in both groups), despite patients in the FOLFIRINOX group being significantly younger. ${ }^{27}$ In addition, a retrospective analysis $(\mathrm{N}=150)$ of patients treated at 5 cancer centers in British Columbia, Canada, found that both $n a b$-P/Gem and FOLFIRINOX produced similar outcomes and demonstrated longer OS versus Gem alone as treatment for unresectable PC (median, 11.6 and 11.2 vs 4.1 months, respectively; $P<0.001$ and $P=0.039) .{ }^{29}$ Patients who received FOLFIRINOX were younger (median age, 61 vs 70 years) and fitter (ECOG PS $\leq 1,91 \%$ vs 54\%) than those who received $n a b-\mathrm{P} / \mathrm{Gem} .{ }^{29}$ Collectively, the OS with nab-P/Gem observed in MPACT was consistent with the OS observed in real-world observational data sets, and nab-P/Gem was comparable in effectiveness to FOLFIRINOX.

\section{Subsequent therapies after first-line nab-P/Gem in MPC}

Many recent analyses have examined the use of second-line therapies after $n a b-\mathrm{P} / \mathrm{Gem} .{ }^{27,30-33}$ Patients in MPACT who received second-line therapy $(\mathrm{n}=170)$ after $n a b-\mathrm{P} / \mathrm{Gem}$ experienced a numerically longer median OS than those who did not ( $\mathrm{n}=250$; median total OS, 12.8 and 6.3 months, respectively). ${ }^{30}$ The longest total OS values were observed in patients who received first-line $n a b-\mathrm{P} / \mathrm{Gem}$ followed by fluoropyrimidine-containing second-line regimens $(n=132$; median, 13.5 months); a small number $(n=18)$ received second-line FOLFIRINOX and experienced a median total OS of 15.7 months. ${ }^{30}$ Another retrospective analysis from the previously described Italian registry $(\mathrm{N}=250)$ demonstrated similar findings, that is, a median OS of 13.5 months in patients who received second-line treatment after firstline $n a b$-P/Gem $(\mathrm{n}=122) .{ }^{31}$ More specifically, patients who received second-line FOLFOX/XELOX $(n=56)$, FOLFIRI $(n=24)$, and FOLFIRINOX $(n=22)$ had median total OS values of $12.8,13.2$, and 13.8 months, respectively. ${ }^{31}$ Consistent 
findings have been observed in many other analyses, and the totality of data suggests that first-line $n a b$-P/Gem followed by second-line therapy, particularly with regimens that contain a fluoropyrimidine, is feasible and beneficial to patients with advanced $\mathrm{PC} .{ }^{27,30-33}$

\section{Future directions}

Future directions for $n a b-\mathrm{P} / \mathrm{Gem}$ include studies in which the regimen has been used as a backbone therapy (ie, with another agent) in MPC (Table 3) and as a doublet in locally advanced pancreatic cancer (Table 4). Table 5 displays a list of selected ongoing trials of $n a b-\mathrm{P} / \mathrm{Gem}$ with or without other agents as treatment for metastatic, locally advanced, and resectable disease.

\section{nab-P/Gem as a backbone regimen in MPC (studies with results)}

Because $n a b$-P/Gem has demonstrated survival comparable to that with FOLFIRINOX and a more favorable toxicity profile, this regimen is commonly used as a chemotherapy backbone for other agents (Table 3 ). Agents combined with $n a b-\mathrm{P} / \mathrm{Gem}$ are diverse and include cancer stem cell inhibitors (demcizumab, vismodegib, tarextumab, and BBI-608), those with potential immune-modulating activities (indoximod), those directed against tumor stroma (PEGPH20 and 2-0, 3-0 desulfated heparin), chemotherapies (capecitabine \pm cisplatin), hormone therapy (enzalutamide), and others (erlotinib and apatorsen). In 15 studies of patients with MPC treated with $n a b-\mathrm{P} / \mathrm{Gem}$ combined with other agents

Table 3 Studies of nab-P/Gem + another agent for advanced/metastatic pancreatic cancer (no cutoff based on N)

\begin{tabular}{|c|c|c|c|c|c|c|c|c|}
\hline First author, year & $\begin{array}{l}\text { Type of } \\
\text { study }\end{array}$ & $\begin{array}{l}\text { Line of } \\
\text { Tx }\end{array}$ & $\begin{array}{l}\text { Agent combined } \\
\text { with nab-Pa/Gem }\end{array}$ & $\mathbf{N}$ & MPC, \% & $\begin{array}{l}\text { Age, } \\
\text { median, } \\
\text { years }\end{array}$ & PS & $\begin{array}{l}\text { Median OS } \\
(95 \% \mathrm{CI}) \\
\text { months }\end{array}$ \\
\hline Cohen, $2016^{56}$ & $\mathrm{Ph} \mathrm{lb}$ & lst & Erlotinib $^{b}$ & 19 & 63 & 63 & ECOG 0-I & $9.3(3.3-15.4)$ \\
\hline Ko, $2012^{57}$ & $\mathrm{Ph} \mathrm{I}$ & Ist & Capecitabine $^{c}$ & 15 & 100 & 62 & ECOG 0-2 & 7.5 (NR) \\
\hline $\begin{array}{l}\text { De Jesus-Acosta, } \\
2014^{58}\end{array}$ & $\mathrm{Ph} \|$ & Ist & $\begin{array}{l}\text { Vismodegib added in } \\
\text { cycle } 2\end{array}$ & 59 & 100 & 60 & ECOG 0-I & $10(7.3-I I)$ \\
\hline $\begin{array}{l}\text { ALPINE } \\
\text { O'Reilly, 201559 }\end{array}$ & $\mathrm{Ph} \mathrm{lb}$ & lst & Tarextumab & 40 & 100 & 63 & ECOG 0-I & 11.6 \\
\hline Hidalgo, $2016^{60}$ & $\mathrm{Ph} \mathrm{lb}$ & lst & $\begin{array}{l}\text { Demcizumab } \\
\text { Gem + demcizumab } \\
\text { (no nab-P) }\end{array}$ & 56 & 70 & 65 & NR & $\begin{array}{l}\text { I0.I (6.5-16.2) } \\
\text { NR }\end{array}$ \\
\hline \multirow[t]{2}{*}{ Hingorani, $2016^{61-63}$} & $\mathrm{Ph} I I$ & lst & PEGPH20 & 74 & 100 & NR & NR & $\begin{array}{l}\text { I } 2 \text { (high-HA } \\
\text { population) }\end{array}$ \\
\hline & & & nab-P/Gem only & 61 & & & & $\begin{array}{l}9 \text { (high-HA } \\
\text { population) }\end{array}$ \\
\hline \multirow[t]{2}{*}{ O'Reilly, $2016^{64}$} & $\mathrm{Ph} \mathrm{I}$ & lst & Necuparanib & 27 & 100 & 63 (mean) & ECOG 0-I & $\begin{array}{l}\text { I3.I (4.0-I6.6) } \\
\text { for patients who } \\
\text { completed } \geq \text { I dose }\end{array}$ \\
\hline & & & $\begin{array}{l}\text { Gem + necuparanib } \\
\text { (no nab-P) }\end{array}$ & 12 & & & & $\begin{array}{l}10.4(6.1-2 \mid .8) \\
\text { for patients who } \\
\text { completed } \geq 1 \text { dose }\end{array}$ \\
\hline Bhattacharyya, & Inst. & lst & VT-I22CM & 20 & 65 & 62 & Mean ECOG 1.9 & 17.0 \\
\hline $2015^{65}$ & & & nab-P/Gem only & 17 & 76 & 60 & Mean ECOG 2.I & $\begin{array}{l}9.3 \\
(P<0.00 I)\end{array}$ \\
\hline Mahipal, 201566 & $\mathrm{Ph} \mathrm{I}$ & Ist & Enzalutamide & 8 & 100 & 64 & ECOG I & NR \\
\hline Reni, $2014^{67}$ & $\mathrm{Ph} \mathrm{lb}$ & lst & $\begin{array}{l}\text { Capecitabine }+ \\
\text { cisplatin }^{d}\end{array}$ & 24 & NR & 63 & $K P S \leq 80,13 \%$ & NR \\
\hline Sigal, $2013^{68}$ & $\mathrm{Ph} I I$ & lst & $\begin{array}{l}\text { 2-O, 3-O desulfated } \\
\text { heparin }(\mathrm{ODSH})\end{array}$ & 10 & NR & 66 & ECOG 0-I & NR \\
\hline RAINIER & $\mathrm{Ph} I I$ & Ist & Apatorsen & 66 & 100 & 67 & ECOG 0-I & $5.3(3.2-7.2)$ \\
\hline Ko, 201669 & & & nab-P/Gem only & 66 & 100 & 66 & ECOG 0-I & $\begin{array}{l}6.9 \\
(P=N S)\end{array}$ \\
\hline El-Rayes, $2016^{70}$ & $\mathrm{Ph} \mathrm{lb}$ & $\leq 2 \mathrm{nd}$ & BBI-608 & 37 & 100 & 63 & ECOG 0-I & NR \\
\hline Bahary, 2016 & $\mathrm{Ph} \mathrm{lb}$ & lst & Indoximod & 15 & 100 & 68 & $\mathrm{KPS} \geq 70$ & NR \\
\hline Borad, $2016^{72}$ & $\mathrm{Ph} \mathrm{I}$ & lst & Evofosfamide & 19 & 89 & 62 & ECOG 0-I & 14.2 (8.5-19.4) \\
\hline
\end{tabular}

Notes: a nab-P at $125 \mathrm{mg} / \mathrm{m}^{2}$ the first 3 of 4 weeks (qw 3/4) unless otherwise indicated. ${ }^{b}$ nab-P at 75,100 , or $125 \mathrm{mg} / \mathrm{m}^{2}$ qw $3 / 4$. ${ }^{\mathrm{D} D o s e}$ escalation of nab-P from 100 to 150 $\mathrm{mg} / \mathrm{m}^{2}$ on day 4 of a 14 -day cycle. ${ }^{\mathrm{d}}$ nab-P at $100-150 \mathrm{mg} / \mathrm{m}^{2}$ on days I and $\mathrm{I} 4$ every 4 weeks.

Abbreviations: Cl, confidence interval; ECOG, Eastern Cooperative Oncology Group; Gem, gemcitabine; HA, hyaluronan; Inst, institutional analysis; KPS, Karnofsky performance status; MPC, metastatic pancreatic cancer; NR, not reported; nab-P, nanoparticle albumin-bound paclitaxel; NS, not statistically significant; OS, overall survival; $\mathrm{Ph}$, phase; PS, performance status; Tx, treatment. 
(including 10 phase I trials), the median OS ranged from 6.9 to 17 months.

\section{nab-P/Gem as a backbone regimen in MPC (studies without results)}

Thirty ongoing phase II and III trials of nab-P in PC with a sample size of $\geq 100$ were identified, including 16 MPC trials (all first line); most included an additional agent (Table 5). For example, the phase II/III RESOLVE trial $(\mathrm{N}=326)$ is evaluating $n a b-\mathrm{P} / \mathrm{Gem}$, with or without the Bruton tyrosine kinase inhibitor ibrutinib, as first-line treatment of MPC. ${ }^{34}$ Based on promising results from phase I/II trials (Table 3), a phase III trial $(\mathrm{N}=420)$ is investigating PEGPH20 in combination with $n a b-\mathrm{P} / \mathrm{Gem}$ in patients with high levels of hyaluronan, and demcizumab with $n a b-\mathrm{P} / \mathrm{Gem}$ is being evaluated in the phase II YOSEMITE trial $(\mathrm{N}=201) \cdot{ }^{35}$ Another noteworthy ongoing trial is a phase II study $(\mathrm{N}=260)$ of $n a b-\mathrm{P} / \mathrm{Gem}$ plus istiratumab (MM-141; a bispecific antibody

Table 4 Locally advanced and/or earlier-stage pancreatic cancer studies of $\geq 15$ patients that include treatment with nab-P/Gem

\begin{tabular}{|c|c|c|c|c|c|c|c|c|}
\hline \multirow[t]{2}{*}{ First author, year } & \multirow[t]{2}{*}{$\begin{array}{l}\text { Type of } \\
\text { study }\end{array}$} & \multirow[t]{2}{*}{ Regimen $^{a}$} & \multirow[t]{2}{*}{$\mathbf{N}$} & \multirow[t]{2}{*}{ Stage } & \multirow[t]{2}{*}{$\begin{array}{l}\text { Age, } \\
\text { median, } \\
\text { years }\end{array}$} & \multirow[t]{2}{*}{ Response data } & \multicolumn{2}{|c|}{$\begin{array}{l}\text { Resection rate in all } \\
\text { patients/in patients } \\
\text { who underwent } \\
\text { resection }\end{array}$} \\
\hline & & & & & & & Ro & $\mathbf{R} \mathbf{I}$ \\
\hline Sueyoshi, $2015^{51}$ & $\mathrm{Ph} \mathrm{I}$ & $\begin{array}{l}n a b-\mathrm{P}^{\mathrm{b}} / \mathrm{Gem}+ \\
\text { radiation }\end{array}$ & 15 & Unresectable LAPC & 63 & $\begin{array}{l}P R=13 \% \\
S D=67 \% \\
P D=7 \%\end{array}$ & NA & NA \\
\hline Dean, $2016^{52}$ & Retro & $\begin{array}{l}\text { nab-P/Gem } \rightarrow \\
\text { 5-FU CRT }\end{array}$ & 42 & Unresectable LAPC & 66 & $\mathrm{pCR}=33 \%$ & $7 \% / 38 \%$ & $12 \% / 63 \%$ \\
\hline \multirow[t]{2}{*}{ Idrees, $2016^{33}$} & Retro & nab-Pc/Gem & 26 & $\begin{array}{l}\text { BL resectable }(77 \%) \text { and } \\
\text { LAPC }(23 \%)\end{array}$ & NR & $\mathrm{pCR}=15 \%$ & $\begin{array}{l}\text { NR/86\% (not } \\
\text { given for each }\end{array}$ & NR \\
\hline & & FOLFIRINOX & 59 & $\begin{array}{l}\text { BL resectable }(63 \%) \text { and } \\
\text { LAPC }(37 \%)\end{array}$ & NR & $\mathrm{pCR}=5 \%$ & group) & NR \\
\hline Peterson, $2016^{53}$ & Retro & nab-P/Gem & 20 & $\begin{array}{l}\text { BL resectable }(70 \%) \text { and } \\
\text { unresectable }(30 \%) \text {; patients } \\
\text { ineligible for FOLFIRINOX }\end{array}$ & 69 & $P R=20 \%$ & $20 \% / 67 \%$ & NA \\
\hline $\begin{array}{l}\text { NEOPAX, } \\
\text { Van Laethem, } \\
2016^{54}\end{array}$ & $\mathrm{Ph} 0$ & nab-P/Gem & 23 & $\begin{array}{l}\text { Unresectable and } \\
\text { borderline resectable }\end{array}$ & 63 & $\begin{array}{l}P R=35 \% \\
P C R=0\end{array}$ & $30 \% / N R$ & $26 \% / N R$ \\
\hline \multirow[t]{2}{*}{$\begin{array}{l}\text { GAIN-I; Sliesoraitis, } \\
2014^{55}\end{array}$} & $\mathrm{Ph}$ II & nab-Pd/Gem & 10 & $\begin{array}{l}\text { Resectable/borderline } \\
\text { resectable }\end{array}$ & 68 & & $60 \% / 75 \%$ & $20 \% / 25 \%$ \\
\hline & & $\begin{array}{l}\text { Non-neoadjuvant } \\
\text { historic controls }\end{array}$ & 22 & & 67 & & $77 \% / N R$ & $9 \% / N R$ \\
\hline Alvarez, $2013^{40}$ & NR & nab-P/Gem & 16 & $\begin{array}{l}\text { Resectable, } 44 \% \text {; } \\
\text { borderline resectable, } 56 \%\end{array}$ & 58 & $\begin{array}{l}\text { PR by PET, } 50 \% \text {; } \\
\text { no objective } \\
\text { responses; } \\
\text { I complete } \\
\text { pathological } \\
\text { response, } 6 \\
\text { GRT-I, I GRT-2, } \\
2 \text { GRT-3 }\end{array}$ & $69 \% / 92 \%$ & $6 \% / 8 \%$ \\
\hline $\begin{array}{l}\text { GAP; Barbour, } \\
2015^{39}\end{array}$ & $\mathrm{Ph}$ II & nab-P/Gem & 41 & Resectable & 65 & $\begin{array}{l}\text { Pancreatic } \\
\text { resection rate, } \\
73 \%\end{array}$ & $\begin{array}{l}\text { I-mm margin: } \\
37 \% / 52 \% \\
0-\mathrm{mm} \text { margin: } \\
61 \% / 86 \%\end{array}$ & $\begin{array}{l}\text { I-mm } \\
\text { margin: } \\
34 \% / 48 \% \\
0-\mathrm{mm} \\
\text { margin: } \\
10 \% / \mid 4 \%\end{array}$ \\
\hline MacKenzie, $2013^{38}$ & $\mathrm{Ph} I \mathrm{I}$ & nab-P/Gem & 25 & Resectable & 65 & $\begin{array}{l}\text { RECIST } \\
\qquad \begin{array}{l}P R=36 \% \\
S D=18 \% \\
P D=8 \%\end{array}\end{array}$ & $80 \% / 95 \%$ & $4 \% / 5 \%$ \\
\hline
\end{tabular}

Notes: ${ }^{a}$ nab-P at $125 \mathrm{mg} / \mathrm{m}^{2}$ the first 3 of 4 weeks (qw 3/4) unless otherwise indicated. ${ }^{b}$ nab-P at $50-125 \mathrm{mg} / \mathrm{m}^{2}$ qw 3/4. ${ }^{\mathrm{C} D o s e}$ and schedule of nab-P not reported. ${ }^{\mathrm{d}}$ nab-P at $100 \mathrm{mg} / \mathrm{m}^{2}$ qw $3 / 4$.

Abbreviations: 5-FU, 5-fluorouracil; BL, baseline; CRT, chemoradiation therapy; FOLFIRINOX, folinic acid, 5-fluorouracil, irinotecan, and oxaliplatin; Gem, gemcitabine; GRT, grade of residual tumor; LAPC, locally advanced pancreatic cancer; NA, not applicable; NR, not reported; nab-P, nanoparticle albumin-bound paclitaxel; pCR, pathological complete response; PET, positron emission tomography; Ph, phase; PD, progressive disease; PR, partial response; RECIST, Response Evaluation Criteria In Solid Tumors; Retro, retrospective; SD, stable disease. 
against ErbB3 and insulin-like growth factor-1 [IGF-1] receptor) for the first-line treatment of patients with MPC and high serum levels of free IGF-1. ${ }^{36}$ Finally, whether the combination of $n a b-\mathrm{P} / \mathrm{Gem}$ with checkpoint inhibitors will be an effective strategy for PC is an important question, because checkpoint inhibitors have recently provided breakthrough treatment options for several tumor types and are currently being explored in a number of PC trials. Data on such combinations (eg, nab-P/Gem and nivolumab) ${ }^{37}$ are preliminary at this point.

\section{Neoadjuvant trials for patients with resectable, borderline resectable, or LAPC (studies with results)}

Several recent studies $(n=12)$ examined neoadjuvant $n a b-\mathrm{P} / \mathrm{Gem}$ as a strategy for improving $\mathrm{R} 0$ resection rates in resectable tumors or converting borderline resectable tumors to resectable tumors. One of the main pathologic predictors of survival after surgery is resection margin status; a negative resection margin $(\mathrm{R} 0)$ is associated with better prognosis compared with a positive margin. Eight of the 12 studies had a total enrollment of $\geq 15$ patients (Table 4 ). Noteworthy among these is a pilot phase II study in which patients with resectable PC $(\mathrm{N}=25)$ were treated with neoadjuvant $n a b-\mathrm{P} / \mathrm{Gem}$ for 3 cycles. ${ }^{38}$ Surgical resection was possible in $84 \%$ of patients and resulted in R0 resection in $95 \%$ of resected cases, or $80 \%$ of the intention-to-treat population. ${ }^{38}$ The phase II GAP study also evaluated neoadjuvant $n a b-\mathrm{P} / \mathrm{Gem}$ for 2 cycles in patients with resectable PC $(\mathrm{N}=41) .{ }^{39}$ After neoadjuvant treatment, $73 \%$ of the patients underwent pancreatic resection. ${ }^{39}$ Similar results were reported from another trial of neoadjuvant $n a b-\mathrm{P} /$ Gem (administered for 2 cycles) in patients with resectable or borderline resectable tumors $(\mathrm{N}=16) .{ }^{40}$ Seventy-five percent of patients underwent surgery, and $\mathrm{R} 0$ resection was achieved in $69 \%$ of the intention-to-treat population $-92 \%$ of those who underwent surgery.

Table 5 Selected ongoing phase II/III trials $(\mathrm{N} \geq 100)$ of nab-P/Gem \pm other agents in pancreatic adenocarcinoma

\begin{tabular}{|c|c|c|c|c|c|}
\hline Trial & Phase & $\begin{array}{l}\text { Planned } \\
\mathbf{N}\end{array}$ & $\begin{array}{l}\text { Patient population } \\
\text { or stage of disease }\end{array}$ & Regimen & $\begin{array}{l}\text { Planned primary } \\
\text { endpoints }\end{array}$ \\
\hline \multicolumn{6}{|l|}{$\begin{array}{l}\text { Metastatic or advanced stage } \\
\text { nab-P/Gem only }\end{array}$} \\
\hline QOLINPAC, NCT0210688473 & II & 110 & $\begin{array}{l}\text { Unresectable LAPC } \\
\text { or metastatic }\end{array}$ & First-line $n a b-\mathrm{P} / \mathrm{Gem}$ vs Gem & $\begin{array}{l}\text { Deterioration-free QOL } \\
\text { using EORTC QLQ-C30 }\end{array}$ \\
\hline $\begin{array}{l}\text { ALPACA, } \\
\text { NCT02564I } 46^{74}\end{array}$ & II & 325 & Metastatic & $\begin{array}{l}\text { First-line: induction with } \\
\text { nab-P/Gem } \rightarrow \text { nab-P/Gem vs } \\
\text { induction with nab-P/Gem } \rightarrow \\
\text { nab-P/Gem or alternating Gem } \\
\text { monotherapy and nab-P/Gem }\end{array}$ & OS \\
\hline \multicolumn{6}{|l|}{ nab-P/Gem + other } \\
\hline NCT0210102I 75 & III & 430 & Metastatic & $\begin{array}{l}\text { First-line nab-P/Gem }+ \\
\text { momelotinib vs nab-P/Gem }\end{array}$ & DLT, OS \\
\hline NCT027I $5804^{76}$ & III & 420 & Metastatic & $\begin{array}{l}\text { First-line nab-P/Gem }+ \\
\text { PEGPH20 vs nab-P/Gem }+ \\
\text { placebo }\end{array}$ & PFS, OS \\
\hline RESOLVE, NCT02436668 & $\| / I I I$ & 326 & Metastatic & $\begin{array}{l}\text { First-line nab-P/Gem + } \\
\text { lbrutinib vs nab-P/Gem + } \\
\text { placebo }\end{array}$ & PFS \\
\hline $\begin{array}{l}\text { CARRIE, } \\
\text { NCT0239913736 }\end{array}$ & II & 260 & Metastatic & $\begin{array}{l}\text { First-line nab-P/Gem + } \\
\text { MM-|4I vs nab-P/Gem + } \\
\text { placebo }\end{array}$ & PFS \\
\hline YOSEMITE, NCT0228989835 & II & 201 & Metastatic & $\begin{array}{l}\text { First-line nab-P/Gem + placebo } \\
\text { vs nab-P/Gem + demcizumab } \\
\text { + placebo (truncated course of } \\
\text { demcizumab) vs nab-P/Gem + } \\
\text { demcizumab }\end{array}$ & PFS \\
\hline NCT0255199|77 & II & 168 & Metastatic & $\begin{array}{l}\text { First-line nab-P/Gem vs } \\
\text { nal-IRI + 5-FU + folinic acid vs } \\
\text { nal-IRI + 5-FU + folinic acid + } \\
\text { oxaliplatin }\end{array}$ & PFS \\
\hline $\begin{array}{l}\text { FIRGEMAX, } \\
\text { NCT0282720I78 }\end{array}$ & II & 124 & Metastatic & $\begin{array}{l}\text { First-line nab-P/Gem } \\
\text { alternating with FOLFIRI.3 vs } \\
\text { nab-P/Gem }\end{array}$ & PFS at 6 months \\
\hline
\end{tabular}

(Continued) 
Table 5 (Continued)

\begin{tabular}{|c|c|c|c|c|c|}
\hline Trial & Phase & $\begin{array}{l}\text { Planned } \\
\text { N }\end{array}$ & $\begin{array}{l}\text { Patient population } \\
\text { or stage of disease }\end{array}$ & Regimen & $\begin{array}{l}\text { Planned primary } \\
\text { endpoints }\end{array}$ \\
\hline SEQUENCE, NCT0250433379 & $\mathrm{I} / \mathrm{II}$ & 180 & Metastatic & $\begin{array}{l}\text { First-line nab-P/Gem } \rightarrow \\
\text { recommended dose of } \\
\text { modified FOLFOX from } \\
\text { phase I }\end{array}$ & $\begin{array}{l}\text { Phase I: safety, DLT } \\
\text { Phase II: OS at I } 2 \text { months }\end{array}$ \\
\hline PACT-19, NCT0I730222 ${ }^{80}$ & $\mathrm{I} / \mathrm{II}$ & 134 & Advanced & $\begin{array}{l}\text { Phase II: first-line nab-P RP2D } \\
+ \text { Gem } 800 \mathrm{mg} / \mathrm{m}^{2}+\text { cisplatin } \\
30 \mathrm{mg} / \mathrm{m}^{2}+\text { cape I,250 mg/m² } \\
\text { q2w every } 4 \text { weeks vs nab-P } \\
125 \mathrm{mg} / \mathrm{m}^{2}+\text { Gem I,000 } \\
\mathrm{mg} / \mathrm{m}^{2} \text { qw } 3 / 4\end{array}$ & $\begin{array}{l}\text { Phase I: DLT } \\
\text { Phase II: PFS for stage } \\
\text { IV, resectability rate for } \\
\text { stage III }\end{array}$ \\
\hline $\begin{array}{l}\text { NabucCO, } \\
\text { NCT02 } 109341^{81}\end{array}$ & $\mathrm{I} / \mathrm{II}$ & 114 & Metastatic & $\begin{array}{l}\text { First-line nab-P + FOLFIRI or } \\
n a b-P+F O L F O X\end{array}$ & MTD, DLTs, ORR \\
\hline NCT0219482982 & $\mathrm{I} / \mathrm{II}$ & 133 & Advanced & $\begin{array}{l}\text { First-line nab-P/Gem } \pm \\
\text { MK-I } 775\end{array}$ & $\begin{array}{l}\text { Phase I: MTD } \\
\text { Phase II: PFS }\end{array}$ \\
\hline \multicolumn{6}{|l|}{$\begin{array}{l}\text { Resectable or locally advanced } \\
\text { nab-P/Gem only }\end{array}$} \\
\hline LAPACT, NCT0230I I 4344,83 & II & 110 & Untreated LAPC & $n a b-P / G e m$ & Time to treatment failure \\
\hline APACT, NCTOI $964430^{45}$ & III & 800 & Resected & Adjuvant nab-P/Gem vs Gem & DFS \\
\hline NEONAX, NCT020475 I $3^{47}$ & II & 166 & Resectable & $\begin{array}{l}\text { Neoadjuvant and adjuvant vs } \\
\text { only adjuvant nab-P/Gem }\end{array}$ & Time to DFS \\
\hline $\begin{array}{l}\text { SI505, } \\
\text { NCT02562716 } 6^{84}\end{array}$ & II & 112 & Resectable & $\begin{array}{l}\text { Neoadjuvant nab-P/Gem vs } \\
\text { mFOLFIRINOX }\end{array}$ & OS \\
\hline NCT02506842 ${ }^{48}$ & III & 300 & Resected & $\begin{array}{l}\text { Second-line adjuvant } \\
\text { nab-P } 100 \mathrm{mg} / \mathrm{m}^{2}+\text { Gem } \\
\mathrm{I}, 000 \mathrm{mg} / \mathrm{m}^{2} \mathrm{vs} \text { oxaliplatin }+ \\
\text { folinic acid + 5-FU }\end{array}$ & OS \\
\hline NCT02243007 & II & 112 & Resectable & $\begin{array}{l}\text { Neoadjuvant FOLFIRINOX vs } \\
\text { nab-P/Gem }\end{array}$ & OS at 18 months \\
\hline \multicolumn{6}{|l|}{ nab-P/Gem + other } \\
\hline NEOLAP, NCT021 $25136^{41}$ & II & 168 & $\begin{array}{l}\text { Untreated } \\
\text { unresectable or } \\
\text { borderline resectable } \\
\text { LAPC }\end{array}$ & $\begin{array}{l}\text { Neoadjuvant nab-P/Gem } \\
\text { vs nab-P/Gem followed by } \\
\text { FOLFIRINOX }\end{array}$ & $\begin{array}{l}\text { Conversion rate to } \\
\text { resection }\end{array}$ \\
\hline $\begin{array}{l}\text { “Personalized Medicine," } \\
\text { NCT0I72658285 }\end{array}$ & II & 120 & $\begin{array}{l}\text { Resectable and } \\
\text { borderline resectable }\end{array}$ & $\begin{array}{l}\text { nab-P/Gem } \pm \text { subsequent } \\
\text { CRT with Gem or } \\
\text { cape as neoadjuvant or } \\
\text { adjuvant therapy vs other } \\
\text { chemotherapies in similar } \\
\text { settings vs CRT with Gem or } \\
\text { cape in similar settings }\end{array}$ & Resectability rate \\
\hline $\begin{array}{l}\text { SCALOP-2, } \\
\text { NCT0202400986 }\end{array}$ & $\mathrm{I} / \mathrm{II}$ & 289 & LAPC & $\begin{array}{l}\text { Induction nab-P/Gem } \rightarrow \\
\text { nab-P/Gem }+\mathrm{RT} \rightarrow \text { cape }+ \\
\mathrm{RT} \pm \text { nelfinavir vs } 6 \text { cycles of } \\
\text { nab-P/Gem }\end{array}$ & OS, PFS \\
\hline
\end{tabular}

Abbreviations: 5-FU, 5-fluorouracil; cape, capecitabine; CRT, chemoradiation therapy; DFS, disease-free survival; DLT, dose-limiting toxicity; EORTC, European Organisation for Research and Treatment of Cancer; FOLFIRI, folinic acid, 5-fluorouracil, and irinotecan; FOLFIRINOX, folinic acid, 5-fluorouracil, irinotecan, and oxaliplatin; FOLFOX, folinic acid, 5-fluorouracil, and oxaliplatin; Gem, gemcitabine; LAPC, locally advanced pancreatic cancer; MTD, maximum tolerated dose; nal-IRI, nanoliposomal irinotecan; nab-P, nanoparticle albumin-bound paclitaxel; OS, overall survival; PFS, progression-free survival; QOL, quality of life; qw 3/4, first 3 of 4 weeks; RP2D, recommended phase II dose; RT, radiotherapy.

\section{Neoadjuvant trials for patients with resectable, borderline resectable, or LAPC (studies without results)}

The phase II NEOLAP trial ( $\mathrm{N}=168)$ will examine the ability of neoadjuvant $n a b-\mathrm{P} / \mathrm{Gem}$ versus FOLFIRINOX to convert unresectable LAPC or borderline resectable tumors to resectable tumors (Table 5). ${ }^{41}$ Another phase II study $(\mathrm{N}=112)$ is comparing neoadjuvant $n a b$ - $\mathrm{P} / \mathrm{Gem}$ versus FOLFIRINOX followed by resection in patients with potentially resectable tumors. ${ }^{42}$ The randomized phase II LAPACT study $(\mathrm{N}=110)$ is investigating time to treatment failure in patients with unresectable LAPC treated with $n a b-\mathrm{P} / \mathrm{Gem} .{ }^{43,44}$ 


\section{Ongoing adjuvant trials for patients with resectable PC}

The ongoing phase III APACT study is evaluating $n a b-\mathrm{P} / \mathrm{Gem}$ versus Gem monotherapy as adjuvant treatment in patients who have undergone macroscopic complete resection for non-MPC (Table 5) ${ }^{45,46}$ Two other studies are also examining $n a b-\mathrm{P} / \mathrm{Gem}$ as adjuvant therapy: the phase II NEONAX study $(\mathrm{N}=166 ; n a b-\mathrm{P} / \mathrm{Gem}$ as adjuvant only vs as neoadjuvant plus adjuvant ${ }^{47}$ and a second-line adjuvant phase III trial in patients who experienced disease relapse during Gem-based adjuvant therapy $(\mathrm{N}=300) .^{48}$

\section{Discussion}

Multiple studies have demonstrated that first-line treatment with $n a b-\mathrm{P} / \mathrm{Gem}$ improves survival in patients with MPC, with OS similar to or better than that observed in MPACT. These studies have helped to confirm the dose and schedule of $n a b-\mathrm{P} 125 \mathrm{mg} / \mathrm{m}^{2}$ plus Gem $1,000 \mathrm{mg} /$ $\mathrm{m}^{2} \mathrm{qw} 3 / 4$ as an effective and tolerable option for patients with MPC. Retrospective analyses of comparisons between $n a b$-P/Gem and FOLFIRINOX suggested similar efficacy outcomes between the regimens, despite differences in patient populations; $n a b-\mathrm{P} / \mathrm{Gem}$ was used in a broader spectrum of patients.

Most studies demonstrated an OS benefit with $n a b-\mathrm{P} / \mathrm{Gem}$ regardless of age group; similarly, patients seem to derive substantial clinical benefit from $n a b-\mathrm{P} / \mathrm{Gem}$ regardless of PS. The demonstrated efficacy of first-line $n a b-\mathrm{P} / \mathrm{Gem}$ has led to a number of studies examining regimens afterward as second-line therapy..$^{27,30-33}$ These studies showed that second-line treatment after $n a b-\mathrm{P} / \mathrm{Gem}$ is feasible and that fluoropyrimidine-containing regimens, and not exclusively FOLFIRINOX, are appropriate options in this setting.

There are currently $>100$ ongoing trials (combined target enrollment $>9,500$ patients) assessing different $n a b-\mathrm{P}$ regimens for the treatment of $\mathrm{PC}$, and these studies will provide critical information regarding optimal combinations for specific patient populations. ${ }^{49}$

\section{Conclusion}

In summary, $n a b-\mathrm{P} / \mathrm{Gem}$ is an effective and well-tolerated regimen for patients with PC. Ongoing trials will evaluate $n a b-\mathrm{P}$ in all stages of PC. The combination of $n a b-\mathrm{P} / \mathrm{Gem}$ has become a standard of care for MPC and a backbone onto which novel therapies are added in ongoing trials. Future directions in this field will revolve around improving our understanding of PC, including its molecular biology, and identifying subsets of patients that may benefit from specific treatments.

\section{Acknowledgments}

Medical writing assistance was provided by John McGuire, $\mathrm{PhD}$, MediTech Media, Ltd, funded by Celgene Corporation. The author is fully responsible for content and editorial decisions for this manuscript. The author is on the speaker's bureau and is a consultant for Celgene Corporation.

\section{Disclosure}

The author reports no other conflicts of interest in this work.

\section{References}

1. American Cancer Society, editor. Cancer Facts and Figures 2016. Atlanta, GA: American Cancer Society; 2016.

2. Surveillance, Epidemiology, and End Results Program. SEER stat facts sheets: pancreas cancer; updated 2016. Available from: http://seer. cancer.gov/statfacts/html/pancreas.html. Accessed September 15, 2016.

3. Ciliberto D, Botta C, Correale P, et al. Role of gemcitabine-based combination therapy in the management of advanced pancreatic cancer: a meta-analysis of randomised trials. Eur J Cancer. 2013;49(3):593-603.

4. Conroy T, Desseigne F, Ychou M, et al. FOLFIRINOX versus gemcitabine for metastatic pancreatic cancer. N Engl J Med. 2011;364(19): 1817-1825.

5. Goldstein D, El-Maraghi RH, Hammel P, et al. nab-Paclitaxel plus gemcitabine for metastatic pancreatic cancer: long-term survival from a phase III trial. J Natl Cancer Inst. 2015;107(2):1-10.

6. NCCN Clinical Practice Guidelines in Oncology. Pancreatic Adenocarcinoma. V1. 2016. Available form: https:/www.nccn.org/professionals/ physician_gls/pdf/pancreatic.pdf. Accessed August 1, 2016.

7. Ducreux M, Cuhna AS, Caramella C, et al. Cancer of the pancreas: ESMO clinical practice guidelines for diagnosis, treatment and followup. Ann Oncol. 2015;26(Suppl 5):v56-v68.

8. Balaban EP, Mangu PB, Khorana AA, et al. Locally advanced, unresectable pancreatic cancer: American Society of Clinical Oncology Clinical Practice Guideline. J Clin Oncol. 2016;34(22):2654-2668.

9. Abrams TA, Meyer G, Moloney J, et al. Patterns of chemotherapy (CT) use in a population-based US-wide cohort of patients (pts) with metastatic pancreatic cancer (MPC). Poster presented at: ASCO 2014 Annual Meeting [abstract 4131].

10. Von Hoff DD, Ramanathan RK, Borad MJ, et al. Gemcitabine plus nab-paclitaxel is an active regimen in patients with advanced pancreatic cancer: a phase I/II trial. J Clin Oncol. 2011;29(34):4548-4554.

11. Cartwright TH, Ginsburg A, Wilfong LS, Harrell RK, Hoverman JR. Use of first-line chemotherapy for advanced pancreatic cancer: FOLFIRINOX versus gemcitabine-based therapy. Poster presented at: ASCO 2014 Annual Meeting [abstract 4132].

12. Santoni M, Bittoni A, Andrikou K, et al. Does first-line therapy affect the outcome of patients with pancreatic cancer? Poster presented at: ESMO 2014 Annual Meeting [abstract 695P].

13. Krishna K, Blazer M, Wei L, et al. Modified gemcitabine and nabpaclitaxel in patients with metastatic pancreatic cancer (MPC): a single institution experience. Poster presented at: ASCO 2015 Gastrointestinal Cancers Symposium [abstract 366].

14. Giordano G, Febbraro A, Vaccaro V, et al. Nab-paclitaxel (nab-P) and gemcitabine (G) as first line chemotherapy (CT) in advanced pancreatic cancer (APDAC) patients (pts): an Italian "real life" study. Poster presented at: ESMO 2015 Annual Meeting [abstract 2334].

15. Hammel P, Bachet JB, Desrame J, et al. Nab-paclitaxel plus gemcitabine or plus simplified LV5FU2 as first-line therapy in patients with metastatic pancreatic adenocarcinoma: a GERCOR randomized phase II study. Poster presented at: ASCO 2016 Annual Meeting [abstract 4120].

16. Zhang DS, Wang DS, Wang ZQ, et al. Phase I/II study of albuminbound nab-paclitaxel plus gemcitabine administered to Chinese patients with advanced pancreatic cancer. Cancer Chemother Pharmacol. 2013;71(4):1065-1072. 
17. Shen L, Yu X, Hao J, et al. A phase II study of Chinese patients (pts) treated with $n a b$-paclitaxel (nab-P) plus gemcitabine (gem) for metastatic pancreatic cancer (MPC). Poster presented at: ASCO 2016 Annual Meeting [abstract 327].

18. Ueno H, Ikeda M, Ueno M, et al. Phase I/II study of nab-paclitaxel plus gemcitabine for chemotherapy-naive Japanese patients with metastatic pancreatic cancer. Cancer Chemother Pharmacol. 2016;77(3):595-603.

19. Kasuga A, Ueno H, Ikeda M, et al. Efficacy, safety and pharmacokinetics of weekly nab-paclitaxel plus gemcitabine in Japanese patients with metastatic pancreatic cancer (MPC): phase I/II trial. Presentation at the 2014 APA/JPS Meeting [abstract 14200].

20. Bachet J, Hammel P, Desrame J, et al. Nab-paclitaxel plus gemcitabine or plus simplified LV5FU2 as first-line therapy in patients with metastatic pancreatic adenocarcinoma. A GERCOR randomized phase II study (AFUGEM). Poster presented at: ESMO 2015 Annual Meeting [abstract P317].

21. Lo Re G, Santeufemia DA, Foltran L, Bidoli E, Basso SM, Lumachi F. Prognostic factors of survival in patients treated with nab-paclitaxel plus gemcitabine regimen for advanced or metastatic pancreatic cancer: a single institutional experience. Oncotarget. 2015;6(10):8255-8260.

22. Giordano G, De Vita F, Melisi D, et al. Analysis of activity, efficacy and safety of first line nab-paclitaxel (nab-P) and gemcitabine (G) in advanced pancreatic cancer (APDAC) frail and elderly patients (pts). Poster presented at: ESMO 2015 Annual Meeting [abstract P297].

23. Scheithauer W, Von Hoff DD, Ramanathan R, et al. Dose delivery in a phase III trial (MPACT) of weekly nab-paclitaxel (nab-P) plus gemcitabine (gem) vs gem alone for patients with metastatic adenocarcinoma of the pancreas. Poster presented at: ESMO 2013 Annual Meeting [abstract 2.586].

24. Guillen-Ponce C, Lopez R, Macarulla T, et al. A phase I/II trial to evaluate the efficacy and safety of nab-paclitaxel in combination with gemcitabine for the treatment of frail patients with advanced or metastatic pancreatic cancer: safety results of the phase I trial. Poster presented at: ESMO 2014 Annual Meeting [abstract 700].

25. Montes AF, Villarroel PG, Ayerbes MV, et al. Retrospective analysis of prognostic and predictive markers in patients with locally advanced unresectable or metastatic pancreatic adenocarcinoma treated with gemcitabine/nabpaclitaxel: influence of the presence of stent. Poster presented at: ASCO 2015 Annual Meeting [abstract 483].

26. Park PYS, McAferty KV, Ahmadi M, et al. Radiological markers of treatment responsiveness in patients (pts) with metastatic pancreatic ductal adenocarcinomas (mPDAC) receiving systemic chemotherapy. Poster presented at: ASCO 2016 Annual Meeting [abstract 403].

27. Braiteh FS, Patel M, Parisi M, Ni Q, Park SY, Faria C. Comparative effectiveness and resource utilization of $n a b$-paclitaxel plus gemcitabine $(n a b-\mathrm{P}+\mathrm{G})$ versus FOLFIRINOX (FFX) in first-line treatment of advanced pancreatic adenocarcinoma (PDAC) in a U.S. community oncology setting. Poster presented at: ASCO 2016 Annual Meeting [abstract 433].

28. Braiteh FS, Patel M, Parisi M, Ni Q, Park SY, Faria C. Comparative effectiveness and resource utilization of nab-paclitaxel plus gemcitabine (nab-P+G) versus gemcitabine monotherapy $(\mathrm{G})$ in first-line treatment of advanced pancreatic adenocarcinoma (PDAC) in a U.S. community oncology setting. Poster presented at: ASCO 2016 Annual Meeting [abstract 429].

29. Wang Y, Chen L, Camateros P, Gill S, Renouf DJ, Cheung WY. Comparative effectiveness of FOLFIRINOX or nab-paclitaxel plus gemcitabine in locally advanced or metastatic pancreatic cancer: a population-based analysis. Poster presented at: ASCO 2016 Annual Meeting [abstract 6561].

30. Chiorean EG, Von Hoff DD, Tabernero J, et al. Second-line therapy after nab-paclitaxel plus gemcitabine or after gemcitabine for patients with metastatic pancreatic cancer. Br J Cancer. 2016;115(2):188-194.

31. Giordano G, Febbraro A, Milella M, et al. Impact of second-line treatment (2L T) in advanced pancreatic cancer (APDAC) patients (pts) receiving first line nab-paclitaxel (nab-P) + gemcitabine (G): an Italian multicentre real life experience. Poster presented at: ASCO 2016 Annual Meeting [abstract 4124].
32. Schmidt SL, Durkal V, Jayavalsan SP, et al. Outcomes in metastatic pancreatic adenocarcinoma (MPAC) patients treated with FOLFIRINOX (FFX)/FOLFOX(FX) and gemcitabine + nab-paclitaxel (NabG). Poster presented at: ASCO 2016 Annual Meeting [abstract 397].

33. Idrees K, Parikh A, Postlewait LM, et al. Treatment of borderline resectable (BR) and locally advanced (LA) pancreatic cancer in the era of FOLFIRINOX and gemcitabine plus nab-paclitaxel: a multi-institutional study. Poster presented at: ASCO 2016 Annual Meeting [abstract 451].

34. ClinicalTrials.gov. Study of ibrutinib vs placebo, in combination with nab-paclitaxel and gemcitabine, in the first line treatment of patients with metastatic pancreatic adenocarcinoma (RESOLVE). Available from: https://clinicaltrials.gov/ct2/show/NCT02436668. Accessed August 1, 2015.

35. ClinicalTrials.gov. Study of gemcitabine, ABRAXANE ${ }^{\circledR}$ plus placebo versus gemcitabine, $\mathrm{ABRAXANE}^{\circledR}$ plus 1 or 2 truncated courses of demcizumab in subjects with 1st-line metastatic pancreatic ductal adenocarcinoma (YOSEMITE). Available from: https://clinicaltrials. gov/ct2/show/NCT02289898. Accessed August 1, 2016.

36. ClinicalTrials.gov. A phase 2 study of MM-141 plus nab-paclitaxel and gemcitabine in front-line metastatic pancreatic cancer (CARRIE). Available from: https://clinicaltrials.gov/ct2/show/NCT02399137. Accessed August 1, 2016.

37. Hochster HS, Wainberg ZA, Gutierrez M, et al. Phase 1 study of nivolumab + nab-paclitaxel +/- gemcitabine in pancreatic cancer: safety evaluation of patients treated with nivolumab + nab-paclitaxel in arm A. Poster presented at: AACR: Special Conference on Pancreatic Cancer 2016 [abstract A72].

38. MacKenzie S, Zeh H, McCahill L, et al. A pilot phase II multi center study of nab-paclitaxel and gemcitabine as preoperative therapy for potentially resectable pancreatic cancer (PC). Poster presented at: ASCO 2013 Annual Meeting [abstract 4038].

39. Barbour A, O'Rourke N, Chan H, et al. Initial survival outcomes for the AGITP GAP study - a phase II study of perioperataive nab-paclitaxel and gemcitabine for resectable pancreatic ductal adenocarcinoma (PDAC). Poster presented at: ASCO 2016 Annual Meeting [abstract 4105].

40. Alvarez R, Musteanu M, Garcia-Garcia E, et al. Stromal disrupting effects of nab-paclitaxel in pancreatic cancer. Br J Cancer. 2013;109(4): 926-933.

41. ClinicalTrials.gov. Trial to investigate intensified neoadjuvant chemotherapy in locally advanced pancreatic cancer (NEOLAP). Available from: https://clinicaltrials.gov/ct2/show/NCT02125136?term=nab-pac litaxel+AND+pancreatic+AND+adenocarcinoma\&rank=55. Accessed August 1, 2016.

42. ClinicalTrials.gov. Phase II study of preoperative FOLFIRINOX versus gemcitabine/nab-paclitaxel in patients with resectable pancreatic cancer. Available from: https://clinicaltrials.gov/ct2/show/ NCT02243007?term=nab-paclitaxel+AND+pancreatic+AND+adeno carcinoma\&rank=59. Accessed August 1, 2016.

43. ClinicalTrials.gov. First line treatment of patients with metastatic pancreatic adenocarcinoma (GABRINOX). Available from: https:// clinicaltrials.gov/ct2/show/NCT01964287. Accessed August 1, 2016.

44. Philip PA, Lacy J, Dowden SD, et al. LAPACT: an open-label, multicenter phase II trial of $n a b$-paclitaxel (nab-P) plus gemcitabine (gem) in patients (pts) with locally advanced pancreatic cancer (LAPC). Poster presented at: ASCO 2016 Annual Meeting [abstract TPS477].

45. ClinicalTrials.gov. Nab-paclitaxel and gemcitabine vs gemcitabine alone as adjuvant therapy for patients with resected pancreatic cancer (the "apact" study) (apact). Available from: https://clinicaltrials.gov/ ct $2 /$ show/NCT01964430?term $=$ nab-paclitaxel+AND+pancreatic+AN D+adenocarcinoma\&rank=24. Accessed August 1, 2016.

46. Tempero MA, Coussens LM, Fong L, Manges R, Singh P, Li Y. A randomized, multicenter, double-blind, placebo-controlled study of the bruton tyrosine kinase inhibitor, ibrutinib, versus placebo in combination with nab-paclitaxel and gemcitabine in the first-line treatment of patients with metastatic pancreatic adenocarcinoma (RESOLVE). Poster presented at: ASCO 2016 Annual Meeting [abstract TPS483]. 
47. ClinicalTrials.gov. Neoadjuvant plus adjuvant or only adjuvant nab-paclitaxel plus gemcitabine for resectable pancreatic cancer (NEONAX). Available from: https://clinicaltrials.gov/ct2/show/ NCT02047513?term=nab-paclitaxel+AND+pancreatic+AND+adeno carcinoma\&rank=34. Accessed August 1, 2016.

48. ClinicalTrials.gov. Second-line adjuvant therapy with nab-paclitaxel plus gemcitabine versus oxaliplatin plus folinic acid and fluorouracil for gemcitabine-refractory pancreatic cancer after curative resection. Available from: https://clinicaltrials.gov/ct2/show/NCT02506842?term=nab-pac litaxel+AND+pancreatic+AND+adenocarcinoma\&rank=42. Accessed August 1, 2016.

49. ClinicalTrials.gov. 2016. Available from: https://clinicaltrials.gov. Accessed August 1, 2016.

50. Giordano G, Febbraro A, Vaccaro V, et al. Nab-paclitaxel (nab-P) and gemcitabine (G) as first line chemotherapy (CT) in advanced pancreatic cancer (APDAC) elderly patients (pts): an Italian "real life" study. Poster presented at: ESMO 2015 Annual Meeting [abstract P296].

51. Sueyoshi H, Ioka T, Tamura T, et al. Phase I study of chemoradiation therapy (nab-paclitaxel/gemcitabine) in 15 patients with unresectable locally advanced pancreatic cancer (UR-LAPC). Poster presented at: ASCO 2015 Annual Meeting [abstract 475].

52. Dean AP, Spry N, McGrath A. Nab-paclitaxel plus gemcitabine followed by radiotherapy with concurrent 5 -fu in locally advanced unresectable pancreatic cancer: a Western Australian experience. Poster presented at: ASCO 2016 Annual Meeting [abstract 430].

53. Peterson S, Loaiza-Bonilla A, Ben-Josef E, et al. Neoadjuvant nabpaclitaxel and gemcitabine (AG) in borderline resectable (BR) or unresectable (UR) locally advanced pancreatic adenocarcinoma (LAPC) in patients ineligible for FOLFIRINOX. Poster presented at: ASCO 2016 Annual Meeting [abstract 328].

54. Van Laethem J, Bali MA, Borbath I, et al. Preoperative gemcitabinenab-paclitaxel (G-NP) for (borderline) resectable (BLR) or locally advanced (LA) pancreatic ductal adenocarcinoma (PDAC): feasibility results and early response monitoring by diffusion-weighted (DW) MR Poster presented at: ASCO 2016 Annual Meeting [abstract 4116].

55. Sliesoraitis S, Desai NV, Trevino JG, et al. Final results for gemcitabine with nab-paclitaxel in neoadjuvant treatment of resectable pancreatic adenocarcinoma: GAIN-1 study. Poster presented at: ASCO 2014 Annual Meeting [abstract e15201].

56. Cohen SJ, O'Neil BH, Berlin J, et al. A phase 1b study of erlotinib in combination with gemcitabine and nab-paclitaxel in patients with previously untreated advanced pancreatic cancer: an Academic Oncology GI Cancer Consortium study. Cancer Chemother Pharmacol. 2016;77(4):693-701.

57. Ko AH, Truong TG, Kantoff E, et al. A phase I trial of nab-paclitaxel, gemcitabine, and capecitabine for metastatic pancreatic cancer. Cancer Chemother Pharmacol. 2012;70(6):875-881.

58. De Jesus-Acosta A, O'Dwyer PJ, Ramanathan RK, et al. A phase II study of vismodegib, a hedgehog (hh) pathway inhibitor, combined with gemcitabine and nab-paclitaxel (nab-P) in patients (pts) with untreated metastatic pancreatic ductal adenocarcinoma (PDA). Poster presented at: ASCO 2014 Annual Meeting [abstract 257].

59. O'Reilly EM, Smith LS, Bendell JC, et al. Final results of phase Ib of anticancer stem cell antibody tarextumab (OMP-59R5, TRXT, antinotch $2 / 3$ ) in combination with nab-paclitaxel and gemcitabine (nab$\mathrm{P}+\mathrm{Gem}$ ) in patients (pts) with untreated metastatic pancreatic cancer (mPC). Poster presented at: ASCO 2015 Annual Meeting [abstract 278].

60. Hidalgo M, Cooray P, Carrato A, et al. A phase $1 \mathrm{~b}$ study of the anticancer stem cell agent demcizumab (DEM) and gemcitabine (GEM) +/- nab-paclitaxel in patients with pancreatic cancer. Poster presented at: ASCO 2016 Annual Meeting [abstract 341].

61. Hingorani S, Bullock A, Harris W, et al. Final analysis of stage 1 data from a randomized phase 2 study of PEGPH20 plus nab-paclitaxel/ gemcitabine in stage IV previously untreated pancreatic cancer patients, utilizing VENTANA companion diagnostic assay. Poster presented at: ESMO World Congress on Gastrointestinal Cancers 2016 [abstract PD-006].
62. Hingorani SR, Harris WP, Seery TE, et al. Interim results of a randomized phase II study of PEGPH20 added to nab-paclitaxel/gemcitabine in patients with stage IV previously untreated pancreatic cancer. Poster presented at: ASCO 2016 Annual Meeting [abstract 439].

63. Bullock AJ, Hingorani SR, Wu XW, et al. Final analysis of stage 1 data from a randomized phase II study of PEGPH20 plus nab-paclitaxel/ gemcitabine in stage IV previously untreated pancreatic cancer (pts), utilizing VENTANA companion diagnostic assay. Poster presented at: ASCO 2016 Annual Meeting [abstract 4104].

64. O'Reilly EM, Mahalingam D, Roach JM, et al. Safety, pharmacokinetics, pharmacodynamics, and antitumor activity of necuparanib combined with nab-paclitaxel and gemcitabine in patients with metastatic pancreatic cancer: updated phase 1 results. Poster presented at: ASCO 2016 Annual Meeting [abstract 4117].

65. Bhattacharyya GS, Babu KG, Bondarde SA, et al. Effect of coadministered beta blocker and cox-2 inhibitors to patients with pancreatic cancer prior to receiving albumin-bound paclitaxel. Poster presented at: ASCO 2015 Annual Meeting [abstract 302].

66. Mahipal A, Springett GM, Burke N, et al. Phase I trial of enzalutamide in combination with gemcitabine and nab-paclitaxel for the treatment of advanced pancreatic cancer. Poster presented at: ASCO 2015 Annual Meeting [abstract 467].

67. Reni M, Belli C, Balzano G, et al. Phase IB trial of nab-paclitaxel plus gemcitabine, capecitabine, and cisplatin (PAXG regimen) in patients with stage III pancreatic adenocarcinoma. Poster presented at: ESMO World Congress on Gastrointestinal Cancer 2014 [abstract 711P].

68. Sigal D, Marcus SG, Rosen PJ, et al. Association of 2-O, 3-O desulfated heparin $(\mathrm{ODSH})$ plus combination gemcitabine $(\mathrm{G}) /$ nab-paclitaxel (A) with preliminary benefit in untreated metastatic pancreatic cancer plus combination gemcitabine (G)/nab-paclitaxel (A) with preliminary benefit in untreated metastatic pancreatic cancer. Poster presented at: ASCO 2013 Annual Meeting [abstract 284].

69. Ko AH, Murphy PB, Peyton JD, et al. A randomized, double-blinded, placebo-controlled phase II trial of gemcitabine (gem) plus nabpaclitaxel (nab-P) plus apatorsen (A) or placebo (pl) in patients (pts) with metastatic pancreatic cancer (mPC): the RAINIER trial. Poster presented at: ASCO 2016 Annual Meeting [abstract 4119].

70. El-Rayes BF, Shahda S, Starodub A, O'Neil BH, Hanna WT, Oh C. A phase Ib extension study of cancer stemness inhibitor BB608 (napabucasin) in combination with gemcitabine and nab-paclitaxel ( $n a b-\mathrm{PTX)}$ in patients (pts) with metastatic pancreatic cancer. Poster presented at: ASCO 2016 Annual Meeting [abstract 4128].

71. Bahary N, Garrido-Laguna I, Wang-Gillam A, et al. Results of the phase Ib portion of a phase I/II trial of the indoleamine 2,3-dioxygenase pathway (IDO) inhibitor indoximod plus gemcitabine/nab-paclitaxel for the treatment of metastatic pancreatic cancer. Poster presented at: ASCO 2016 Annual Meeting [abstract 452].

72. Borad MJ, Kwak EL, Wang-Gillam A, Ibrahim A, Aldridge J, Olszanski AJ. Evofosfamide combined with gemcitabine/nab-paclitaxel in patients with previously untreated locally advanced or metastatic pancreatic adenocarcinoma (PAC): results of a phase I trial. Poster presented at: ASCO 2016 Annual Meeting [abstract 4114].

73. ClinicalTrials.gov. Quality of life study in patients with locally advanced or metastatic pancreatic cancer treated with gemcitabine in combination with nab-paclitaxel (QOLINPAC). Available from: https:// clinicaltrials.gov/ct $2 /$ show $/ \mathrm{NCT} 02106884$ ?term $=$ nab-paclitaxel+AN $\mathrm{D}+$ pancreatic+AND+adenocarcinoma\&rank $=54$. Accessed August 1 , 2016.

74. ClinicalTrials.gov. First-line treatment of metastatic pancreatic cancer with $n a b$-paclitaxel and gemcitabine (ALPACA). Available from: https:// clinicaltrials.gov/ct2/show/NCT02564146?term=NCT02564146\&r ank=1. Accessed August 1, 2016.

75. ClinicalTrials.gov. Gemcitabine and nab-paclitaxel combined with momelotinib in participants with previously untreated metastatic pancreatic ductal adenocarcinoma. Available from: https://clinicaltrials. gov/ct2/show/NCT02101021. Accessed August 1, 2016. 
76. ClinicalTrials.gov. A study of PEGylated recombinant human hyaluronidase in combination with $n a b$-paclitaxel plus gemcitabine compared with placebo plus nab-paclitaxel and gemcitabine in participants with hyaluronan-high stage IV previously untreated pancreatic ductal adenocarcinoma. Available from: https://clinicaltrials.gov/ct2/show/ NCT02715804. Accessed August 1, 2016.

77. ClinicalTrials.gov. Study of nanoliposomal irinotecan (nal-IRI)containing regimens in patients with previously untreated, metastatic pancreatic adenocarcinoma. Available from: https://clinicaltrials.gov/ ct2/show/NCT02551991. Accessed August 1, 2016.

78. ClinicalTrials.gov. Sequential treatment of nab-paclitaxel + gemcitabine / FOLFIRI.3 vs nab-paclitaxel + gemcitabine in 1st line treatment of metastatic pancreatic cancer (FIRGEMAX). Available from: https://clinicaltrials.gov/ct2/show/NCT02827201. Accessed August 1, 2016.

79. ClinicalTrials.gov. Phase I/II study of nab-paclitaxel and gemcitabine followed by AG-mFOLFOX in patients with metastatic pancreatic adenocarcinoma (SEQUENCE). Available from: https://www.clinicaltrials.gov/ct2/show/NCT02504333. Accessed August 1, 2016.

80. ClinicalTrials.gov. A phase I-II study of PAXG in stage III-IV pancreatic adenocarcinoma (PACT-19). Available from: https:// clinicaltrials.gov/ct2/show/NCT01730222?term=nab-paclitaxel+AN $\mathrm{D}+$ pancreatic $+\mathrm{AND}+$ adenocarcinoma\&rank=12. Accessed August 1, 2016.
81. ClinicalTrials.gov. Phase I/II study to evaluate nab-paclitaxel in substitution of CPT11 or oxaliplatin in FOLFIRINOX schedule as first line treatment in metastatic pancreatic cancer (NabucCO). Available from: https:// clinicaltrials.gov/ct2/show/NCT02109341. Accessed August 1, 2016.

82. ClinicalTrials.gov. Paclitaxel albumin-stabilized nanoparticle formulation and gemcitabine hydrochoride with or without WEE1 inhibitor MK-1775 in treating patients with previously untreated pancreatic cancer that is metastatic or cannot be removed by surgery. Available from: https:// clinicaltrials.gov/ct2/show/NCT02194829. Accessed August 1, 2016.

83. ClinicalTrials.gov. Phase 2 nab $^{\mathrm{TM}}$-paclitaxel (Aabraxane ${ }^{\mathbb{R}}$ ) plus gemcitabine in subjects with locally advanced pancreatic lancer (LAPC) (LAPACT). Available from: http://www.clinicaltrials.gov/ct2/show/ NCT02301143. Accessed August 1, 2016.

84. ClinicalTrials.gov. S1505: combination chemotherapy or gemcitabine hydrochloride and paclitaxel albumin-stabilized nanoparticle formulation before surgery in treating patients with pancreatic cancer that can be removed by surgery. 2016. Available from: https://clinicaltrials.gov/ ct2/show/NCT02562716. Accessed September 15, 2016.

85. ClinicalTrials.gov. Pancreas cancer: molecular profiling as a guide to therapy before and after surgery ("personalized medicine"). Avalable from: https:// clinicaltrials.gov/ct2/show/NCT02562716. Accessed August 1, 2016.

86. ClinicalTrials.gov. Systemic therapy and chemoradiation in advanced localised pancreatic cancer-2 (SCALOP-2). Available from: https:// clinicaltrials.gov/ct2/show/NCT02024009. Accessed August 1, 2016.

\section{Cancer Management and Research}

\section{Publish your work in this journal}

Cancer Management and Research is an international, peer-reviewed open access journal focusing on cancer research and the optimal use of preventative and integrated treatment interventions to achieve improved outcomes, enhanced survival and quality of life for the cancer patient. The manuscript management system is completely online and includes

\section{Dovepress}

a very quick and fair peer-review system, which is all easy to use. Visit http://www.dovepress.com/testimonials.php to read real quotes from published authors. 